\title{
A Systematic Review on Management of Anterior Column Acetabular Fracture
}

M.S.E.Alzahhar, M.G.Montaser, E.A.Tabl and S.A.Abd-Allah

Orthopedic Surgery Dept., Faculty of Medicine, Benha Univ., Benha, Egypt

E-Mail:S.A@gmail.com

\begin{abstract}
\section{Introduction}

Acetabular fractures are rare but significant injuries that involve the articular surface of the acetabulum, with an incidence of three in 100000 per year. These fractures are typically associated with a high-energy mechanism of injury, with half having an injury to another organ system. Historically the most common mechanism is that of a road traffic accident or fall from a height. More recently there has been an increase in low energy fragility acetabular fractures in the elderly caused by falls from standing height. The fracture pattern is determined by the force vector applied across the hip and the position of the femoral head at the time of injury [1].
\end{abstract}

Acetabular fractures are potentially severe fractures associated with high morbidity and disability. Although open reduction and internal fixation are the standard methods of treating displaced acetabular fractures, open reduction has substantial morbidity, such as blood loss, neurovascular injury, wound infection, wound healing problems and heterotopic bone formation. With the development of advanced radiologic technology and new techniques and approaches for reduction and internal fixation, a percutaneous approach for internal fixation has become possible. Percutaneous approach is associated with fewer complications related to surgical exposure. It is a possible treatment option with no or minimal displaced, but instable acetabular fractures with a risk of secondary displacement, and displaced acetabular fractures which can be reduced by cannulate screw.

\section{Material and methods}

A prospective study of surgically treated acetabular fractures

We performed a systematic review of the literature in order to identify articles reporting functional outcomes of surgically managed acetabular fractures. Medline, Google scholar and PubMed were searched for articles published in English up to December 2019 which met the inclusion criteria.

\section{Inclusion criteria}

- Case series with more than three patients.

- Cohort studies.

- Clinical trials reporting functional outcomes after surgically managed acetabular fractures.

- Fractures managed by open reduction and internal fixation (ORIF) within six weeks of injury.

- Anterior column fractures managed by percutaneous cannulated screws or by ORIF by plate and screws.

- Surgical management by an orthopedic surgeon.

- Functional outcomes reported at a minimum three months' follow-up.

- Publication in an orthopedics or trauma journal.

\section{Exclusion criteria}

- Case reports or series with fewer than three patients.

- Studies reporting outcomes of surgery delayed more than six weeks from injury.

- Open fractures of acetabulum.

- Stable posterior wall or column acetabular fractures not requirind surgery.

- Active infection of the hip joint and /or the surrounding soft tissue.

- Patients with severe osteoarthritis hip joint (for THR from the start).

- Non-ambulatory patient.

- Pathologic fractures of acetabulum.

- Acute surgical treatment other than internal fixation by plate and screws or percutaneous fixation by cannulated screws (i.e. immediate total hip arthroplasty).

- Timing of functional outcomes not explicitly stated.

- Review articles, and articles published in a foreign language with no translation available.

The study included patients with anterior acetabular fractures; in all cases the surgery was indicated. There are about 25 studies collected from different institutes and centers specialized in treatment of pelvic fractures. Patients included in the study are of different ages and different methods for treatment.

Total number of patients treated with different methods in our study is 819 patients.

We performed a systematic review of the literature pertaining to the functional outcomes of the surgical management of acetabular fractures. A total of 25 articles met our inclusion criteria, revealing that eight generic outcome instruments were used. The majority of studies reported outcomes using a version of the d'Aubigne and Postel score, which has not been validated for use in acetabular fracture. Few validated outcome measures were reported. No psychometric testing of outcome instruments was performed. The current assessment of outcomes in surgery for acetabular fractures lacks scientific rigour, and scientific 
rigour, and does not give reliable outcome data for either scientific comparison or patient counselling.

Studies included in our study had an associated posterior hemi-transverse fracture that was also managed with minimally invasive fixation. A variety of surgical navigation techniques were used to allow accurate percutaneous screw placement: CT-guided percutaneous fixation was performed; fluoroscopy alone was used and computer-assisted virtual fluoroscopy was used. Some fractures were nondisplaced but potentially unstable, and involved the superior weight-bearing dome; others required closed manipulation using Schanz-pin joysticks.

After confirmation of adequate reduction, largebore cannulated screws were placed percutaneously using previously defined safe trajectories. The quality of fracture reduction and the placement of screw were evaluated by a post-operative $\mathrm{x}$-ray examination.
All patients were managed postoperatively with early mobilization and physical therapy.

\section{Data analysis and statistics}

The gathered data was and entered into Statistical Package of Social Science (SPSS), version 22. Quantitative data were presented by mean and standard deviation or median and interquartile range, while qualitative data were presented by number and percentages. Chi- square and $\mathrm{Z}$ scores were used to compare percentages. The probability of less than 0.05 was used as a cut off point for all significant tests. Figures were done by Excel office 2010 .

\section{Result}

Gender: There are 562 men (69\%) and 257 women $(31 \%)$.

Table (1) Gender.

\begin{tabular}{ll}
\hline Gender & Value \\
\hline Male & $562(69 \%)$ \\
Female & $257(31 \%)$ \\
\hline
\end{tabular}

In our study we do an illustration of number of

fixation by plate and screw, percutaneous fixation every method which used in treatment of anterior column acetabular fracture (open reduction internal

by lag screws or both)

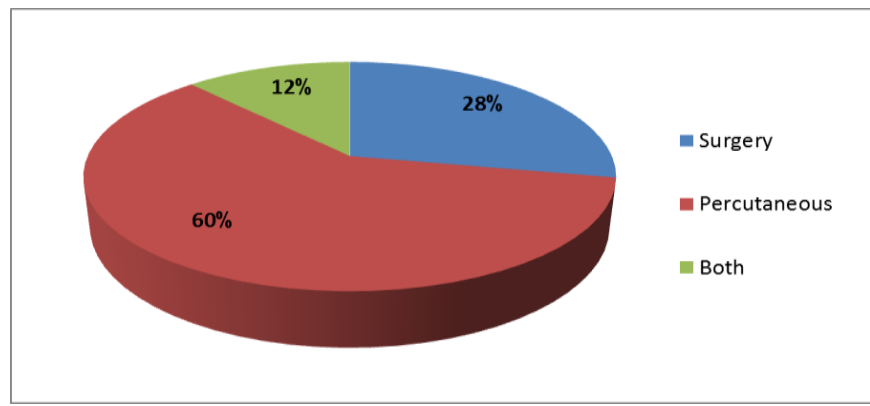

Fig (1) Type of management in studies.

Our study 25 studies collected included 819 patients, there are $60 \%$ of them treated by percutaneous and $40 \%$ of them treated by open reduction and internal fixation.

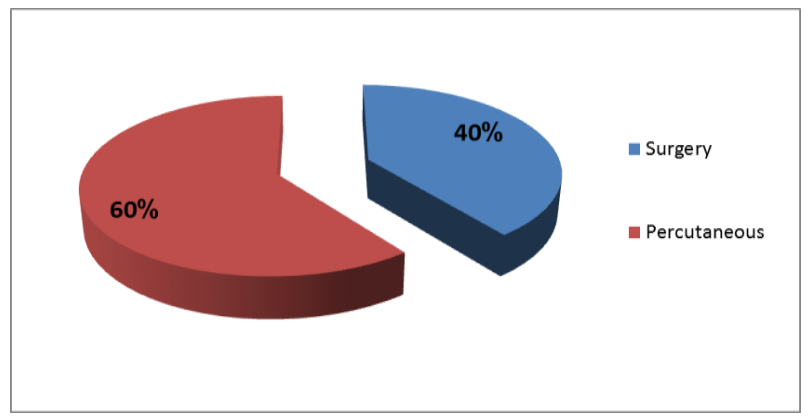

Fig (2) Type of management among patients

In our study, we make a comparison between

the follow up of patients treated by the two 
methods. The reduction of fracture is classified into three categories among all patients that do operative management (anatomical- satisfactory - poor).

\section{Discussion}

In our study, we make a comparison between the follow up of patients treated by open reduction and internal fixation by plate and screws and percutaneous fixation by lag screws. The reduction of fracture is classified into three categories among all patients that do operative management (anatomical- satisfactory - poor).

The study included patients with anterior acetabular fractures; in all cases the surgery was indicated. There are about 25 studies collected from different institutes and centers specialized in treatment of pelvic fracture. Patients included in the study are of different ages and different methods for treatment.

Total number of patients treated with different methods in our study is 728 patients

Reduction and fixation of anterior column by percutaneous screws in our study achieved anatomical reduction in 138 patients (73.8\%); satisfactory in 42 patients $(22.5 \%)$ and poor reduction in 7 patients $(3.7 \%)$ from total 187 patients treated by this method.

While in open reduction and fixation by plate and screws achieved anatomical reduction in 54 patients $(48.6 \%)$, satisfactory in 50 patients $(45 \%)$ and poor in 7 patients $(6.4 \%)$ from total 111 patients treated $b$ this method.

In our study, we made a comparison between complications of each method of management. Complications related to percutaneous fixation include neurological complication in 25 patients $(56.8 \%)$, no pulmonary embolism, deep vein thrombosis in 12 patients $(46.2 \%)$ treated by rivaroxaban, no infection.

While complication related to open reduction include neurological complication in 19 patients (43.2\%), pulmonary embolism in 11 patients, deep vein thrombosis (DVT) in 14 patients and infection in 20 patients.

Intra-operative assessment and postoperative radiological evaluation revealed accurate placement of screws in all of these cases, with no evidence of joint penetration.

In an attempt to overcome the complications of traditional surgical approaches, percutaneous screw fixation has been advocated for the treatment of non-displaced or minimally displaced anterior column fractures of acetabular, and the preliminary results seemed excellent. Though fluoroscopy and three-dimensional reconstruction CT scans can improve the safety and accuracy of the insertion of percutaneous retrograde screw, scholars have realised the risk of cortical penetration in the fixation of the anterior column [1].
Percutaneous stabilization as a minimally invasive treatment option is possible with nondisplaced but instable fractures of the acetabulum with a risk of secondary displacement to allow early mobilization of the patient $[2,3]$.

Moreover, displaced acetabular fractures which can be reduced via lag screw are suitable for percutaneous stabilization. Patients with an elevated perioperative risk from an open approach such as old patients or patients with several injuries in which a reduced approach related morbidity outweighs perfect anatomic fracture reduction, should also be considered for percutaneous stabilization. In addition to this, patients who will need total hip arthroplasty in the short run due to the configuration of the fracture might also benefit from earlier mobilization and protection of soft tissue when undergoing percutaneous stabilization instead of open reduction [4].

Percutaneous screw placement in the anterior column is difficult due to the complex threedimensional pelvic geometry. Despite improved imaging with 3D-CT scans and the intra-operative use of computer assisted navigation, there is a danger of perforation the bone corridor or the acetabulum. Therefore, it is important that the surgeon visualizes the bone stock with the screw corridor in preoperative planning [5].

Internal fixation of acetabular fractures is usually carried out to restore articular congruity of the weight-bearing zone of the hip joint as accurately as possible, thereby attempting to minimize the likelihood of developing subsequent osteoarthritis. Alternatively, in elderly patients, internal fixation can be inserted to provide sufficient stability to the acetabulum to support insertion of a hip replacement [6].

Indications for surgery as with all intra-articular fractures, the goal of acetabular fracture surgery is to reduce each weight-bearing fragment anatomically and achieve stable fixation. Fractures affecting the front of the joint are treated with an anterior approach.

\section{Anterior approaches}

The three most commonly used anterior approaches are described below:

- Modified Stoppa

- Ilioinguinal

- Smith-Peterson.

The Stoppa and ilioinguinal are the anterior 'workhorses' of acetabular fracture surgery. They both expose the anterior column and the quadrilateral. The Smith-Peterson is more distally based and is therefore used to expose the femoral head and neck and the supraacetabular region (anterior inferior iliac spine (AIIS) and anterior acetabular wall [6].

Open reduction with internal fixation is the current standard of care for the treatment of 
displaced acetabular fractures. Accurate reduction and fixation of these articular fractures allows early patient mobilization and minimizes the late complication of post-traumatic arthritis of the hip joint [7].

Classical internal fixation techniques have traditionally required large surgical exposures of the deep structures of the pelvis. These exposures can be associated with significant complications, including infection, wound-healing problems, major blood vessel or nerve injury, denervation or devascularization of the abductors, and heterotopic ossification. The majority of these complications are related to the surgical exposure itself, rather than to the initial traumatic injury. It therefore seems reasonable to consider less invasive alternatives to conventional treatment methods [8].

Recently, closed reduction with percutaneous fixation of nondisplaced or minimally displaced acetabular fractures using computed tomography (CT) or fluoroscopic guidance has attracted interest [9].

Closed reduction with percutaneous fixation of acetabular fractures has the potential to decrease the morbidity associated with traditional open surgical procedures [10].

Virtual fluoroscopy has greatly expanded the potential applications of surgical navigation in routine fracture care. The benefits of computerassisted navigational systems include reduction in radiation exposure to both the patient and surgeon, reduction in procedure times, accurate assessment of alignment, and improved precision in placement of periarticular hardware [11].

The anterior column acetabular fracture is a coronal plane fracture entering the superior weight bearing dome of the acetabulum, and usually exiting directly cephalad to the hip joint, high in the iliac wing. The fracture fragment usually displaces proximally and rotates externally in response to abdominal and gluteal muscle forces. The anterior superior iliac spine (ASIS) is attached to the displaced fragment, and provides convenient access for insertion of a rigid pin to act as a joystick during attempted closed reduction. Following reduction, percutaneous screws may be placed through the anterior inferior iliac spine (AIIS) passing perpendicular to the fracture line, allowing compression of the fracture using lag-screw technique [12].

The objective of surgery for acetabular fractures is to achieve precise reduction to restore joint congruence, fix internal bone fragments, avoid displacement of the fracture and allow rapid rehabilitation. Open reduction and internal fixation is the benchmark method for displaced acetabular fractures, but open reductions can increase morbidity, causing neurovascular injury, blood loss, heterotopic bone formation, infection and poor wound healing [13].

\section{Conclusion}

An anatomical reduction with a gap of $2 \mathrm{~mm}$ or less is a predictor of good joint function and reduced risk of post-traumatic osteoarthritis.

In our study, the percutaneous technique is recommended for nondisplaced or slightly displaced fractures, and in obese, osteoporotic and elderly patients who cannot receive total joint arthroplasty.

We recommend the use of intramedullary cannulated screws.

Fracture reductions are achieved by manual traction of the affected bones. If some fracture displacement remains, accessory windows can be used to introduce a ball spike pusher, a hook or a Steinmann pin which can be used as a joystick to rotate the fracture.

We describe the accessory windows for the anterior column, the quadrilateral plate. We detail the position, direction and kind of screws used to stabilize the anterior columns.

We recommend the use of intramedullary cannulated screws because they resist the rotational, axial and compressive forces within the pelvic ring and help to hold the fracture. Open reduction and definitive internal fixation should always be prepared and planned for as a valid option in case of failed reduction, insufficient stability and redisplacement intra-operatively.

All patients with fractures that have been stabilized by intramedullary means can begin hip mobilization and weight bearing post-operatively. Patients with intramedullary stabilization of the anterior and posterior column can begin walking 48 hours after surgery, with weight bearing of $50 \%$.

\section{Complications and limitations of this technique}

To avoid neurovascular injury and damage to soft tissues, the fracture must be reduced and the intramedullary screw must be carefully introduced into the described corridors, using $\mathrm{C}$-arm imaging to guide progress. This technique is not suitable for comminuted or highly displaced fractures. Surgeons must have experience with percutaneous technique. In future, techniques, such as computer-assisted orthopedic surgery will undoubtedly improve reduction and fixation techniques [14].

Finally, percutaneous intramedullary osteosynthesis, a minimally invasive procedure with low morbidity, should benefit polytrauma patients. Percutaneous treatment can be used in single non-displaced or slightly displaced acetabular fractures. It is not appropriate for comminuted or highly displaced fractures, which must be treated by intrapelvic reduction [15].

\section{References}

[1] S.A.Olson, J.M.Matta, Fractures of the acetabulum, hip dislocations, and femoral head fractures. In: Chapman MW, ed. 
Chapman's orthopaedic surgery. Vol 1. 3rd ed. Philadelphia, Pa: Lippincott Williams \& Wilkins, PP.587-616, 2001.

[2] H.C.Sagi, D.Dziadosz, H.Mir, N.Virani, Obesity, leukocytosis, embolization, and injury severity increase the risk for deep postoperative wound infection after pelvic and acetabular surgery. J Orthop Trauma, Vol.27 (1), PP.6-10, 2013.

[3] M.Vrahas, R.G.Gordon, D.C.Mears, D.Krieger,Intraoperative somatosensory evoked potential monitoring of pelvic and acetabular fractures. J Orthop Trauma.Vol.6(1), PP.50-58, 1992.

[4] B.R.Moed, S.E.Carr, K.I.Gruson, J.T.Watson, Computed tomographic assessment of fractures of the posterior wall of the acetabulum after operative treatment. J Bone Joint Surg Am,Vol.85-A(3), PP.512-522, 2003.

[5] T.J.Blokhuis, J.P.Frölke,Is radiation superior to indomethacin to prevent heterotopic ossification in acetabular fractures? a systematic review. Clin Orthop Relat Res, Vol.467(2), PP.526-530, 2009.

[6] M.Tannast, S.Najibi, JM.Matta, Two to twenty-year survivorship of the hip in 810 patients with operatively treated acetabular fractures. J Bone Jt Surg Am; 94: 1559:67, 2012.

[7] Williams and Wilkins, M.Tile, Fractures of the Pelvis and Acetabulum. 2nd edition. Baltimore, 1995.

[8] FA. Kaempffe, LB. Bone, JR.Border, Open reduction and internal fixation of acetabular fractures: heterotopic ossification and other complications of treatment. J Orthop Trauma , PP.5:439-445, 1991.

[9] GA.Brown, MC.Willis, K.Firoozbakhsh, A.Barmada , Computed tomography image guided surgery in complex acetabular fractures. CORR, PP.370:219-226, 2000.

[10] AL.Ludwig, N.Suhm, A.Kalm, P. Regazzoni et al, Coronal acetabular fractures: the anterior approach in computed tomography-navigated minimally invasive percutaneous fixation. Cardiovasc Intervent Radiol, PP.23:327-331, 2000.

[11] RD.Zura, RD.Kahler, A transverse acetabular nonunion treated with computer-assisted percutaneous internal fixation. J Bone Joint Surg Am, Vol.82, PP.219-224, 2000.

[12] DM.Kahler, R.Zura, Evaluation of a computer integrated surgical technique for percutaneous fixation of transverse acetabular fractures. In: Troccaz J, Grimson E, Mosges $\mathrm{R}$, editors. March 1997. Lecture Notes in Computer Science 1205. Berlin: Springer, P 565-572, 1997.

[13] Lihai Zhang, Wei Zhang, Brian Mullis, Daohong Liu, Qi Xiong, Houchen Lv, Xinran Ji, Ye Peng and Peifu Tang, Percutaneous Anterior Column Fixation for Acetabulum Fractures, Does It Have to Be Difficult?The New Axial Pedicle View of the Anterior Column for Percutaneous Fixation. (J Orthop Trauma; Vol.30, PP.30- 35, 2016).

[14] Y.Peng, L.Zhang, W.Min, P.Tang Comparison of anterograde versus retrograde percutaneous screw fixation of anterior column acetabular fractures. Int J CARS , Vol.11, PP.635-639, 2016.

[15] AJ.Starr, AL.Jones, CM.Reinert, DS.Borer, Preliminary results and complications following limited open reduction and percutaneous screw fixation of displaced fractures of the acetabulum. Injury; 32(suppl 1):SA45-SA50, 2016. 